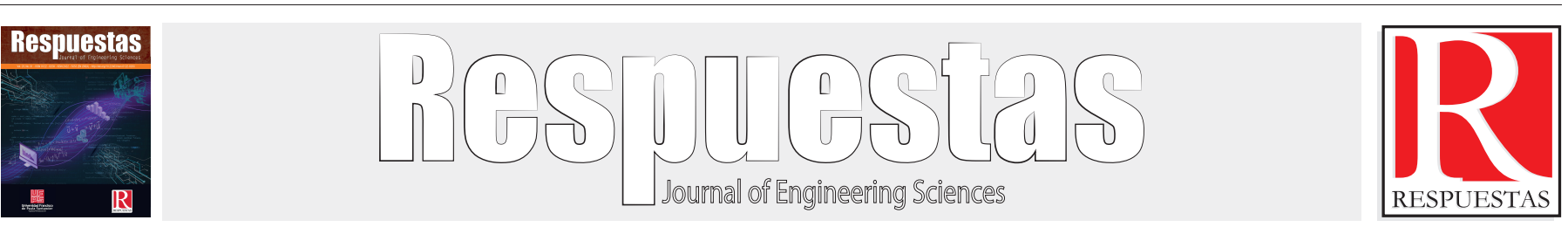

Artículo Original

https://doi.org/10.22463/0122820X.1492

\title{
Generalidades y aporte al estudio de las turbinas tipo Darrieus
}

\section{Generalities and contribution to the study of the Darrieus type turbines}

\author{
Humberto Alejandro Rolón-Ortiza, Carlos Acevedo-Peñaloza,* Yesenia Villamizar-González. \\ aFacultad de ingeniería, humbertoalejandroro@ufps.edu.co, orcid.org/000-0002-8540-2628, (FLUTER), Universidad Francisco de Paula Santander, Cúcuta, Colombia. \\ ${ }^{b}$ Facultad de ingeniería, carloshumbertoap@ufps.edu.co, orcid.org/0000-0002-5049-8754, (GIDIMA), Universidad Francisco de Paula Santander, Cúcuta, Colombia. \\ cFacultad de ingenieria, yeseniavg@ufps.edu.co,orcid.org/0000-0003-4591-9451(FLUTER),Universidad Francisco de Paula Santander, Cúcuta, Colombia.
}

Cómo citar: H.A. Rolón-Ortiz, C. Acevedo-Peñaloza y Y. Villamizar-González, "Modelado matemático y simulación del comportamiento de una turbina hidrocinética tipo darrieus; consideraciones de diseño", Respuestas, vol. 23, no. S1, pp. 14- 18, 2018. https://doi.org/10.22463/0122820X.1492

Recibido: Enero 23, 2018; Aprobado: Junio 29, 2018.

\begin{tabular}{|c|c|}
\hline & RESUMEN \\
\hline \multirow{8}{*}{$\begin{array}{l}\text { Palabras Clave: } \\
\text { CFD } \\
\text { Hidrocinética } \\
\text { Modelo matemático } \\
\text { Simulación }\end{array}$} & $\begin{array}{l}\text { Este trabajo desarrollo el modelamiento y simulación de un rotor Darrieus para conversión de energía hidrocinética. Para } \\
\text { hacerlo, se calculó el porcentaje de energía convertida a partir del modelo de doble disco y múltiples tubos de corriente (DMST). }\end{array}$ \\
\hline & En primer lugar se debió estudiar el perfil aerodinámico de manera individual; para hacerlo, era necesario obtener las curvas de \\
\hline & sustentación y arrastre para posteriormente extrapolarlas a partir del método de extrapolación de Montgomery para lo cual se \\
\hline & desarrolló un programa en MATLAB. Teniendo los datos extrapolados se procedió a realizar un programa que ejecutara el \\
\hline & modelo matemático para obtener la curva Cp Vs TSR, que es la curva característica de los rotores Darrieus, dicho programa se \\
\hline & realizó en MATLAB. Como método de validación de los datos del modelo matemático se optó por hacer una simulación CFD \\
\hline & $\begin{array}{l}\text { en Ansys fluent, se comparó con los trabajos analíticos y experimentales de la bibliografía y finalmente se produjo un prototipo } \\
\text { a escala del rodete. }\end{array}$ \\
\hline & ABSTRACT \\
\hline Keywords: & $\begin{array}{l}\text { This study presents the procedure of modeling and simulation of a Darrieus rotor with the objective of determining the capacity } \\
\text { to take advantage of the energy contained in the water currents for the generation of electrical energy using the model of double }\end{array}$ \\
\hline CFD & disc and multiple stream tubes (DMST). Initially different aerodynamic profiles were analyzed, the profile with the best \\
\hline Hydrokinetics & performance was selected taking into account the coefficients of sustentation and resistance, later these coefficients were \\
\hline Simulation & extrapolated by Montgomery's method using MATLAB software. In this way, the sustentation and resistance coefficients \\
\hline Mathematical model & $\begin{array}{l}\text { corresponding to the established operating conditions were obtained, and then these data were used to develop the characteristic } \\
\text { curve of the Darrieus rotors solving the mathematical model. Finally, the results obtained from the mathematical model were } \\
\text { compared with those obtained through a CFD simulation with the Ansys Fluent software. It was determined that both the } \\
\text { mathematical model and the computational simulation generated results similar to the analytical and experimental studies } \\
\text { performed by other researchers. }\end{array}$ \\
\hline
\end{tabular}

\section{Introducción}

Las turbinas hidrocinéticas son dispositivos que permiten extraer energía a partir de la corriente libre de un cuerpo de agua con cero emisiones contaminantes y bajo impacto en el ecosistema mientras que turbinas Darrieus son un tipo de turbina propuesta en los años 30 por el ingeniero Rudolf Darrieus, se caracteriza por ser de flujo radial y funcionar omnidireccionalmente.

Partiendo del modelo de estudio propuesto por Ion Paraschivoiu [1] para turbinas Darrieus eólicas basado en la teoría del tubo de corriente y el perfil rotativo aislado, encontrando buena correlación con los datos experimentales producidos en el laboratorio SANDIA [2] mejorando las aproximaciones obtenidas con el método del elemento de pala Shiono [3], experimento con prototipos de turbinas Darrieus para corrientes marinas encontrando que el número óptimo de palas debía ser superior a 3 garantizando así el autoarranque. Malipeddi [4] investigó la influencia de ductos en el comportamiento de rotores Darrieus incrementando su eficiencia en $20 \%$.
En este trabajo se analiza el comportamiento de una turbina hidrocinética tipo Darrieus basados en el modelo matemático que delimita el sistema de variables presentes en el fenómeno físico (densidad, velocidad, turbulencia, viscosidad y número de Reynolds), comparándolo con la adecuada simulación. Se calcula el coeficiente de potencia que permita evaluar el desempeño de la turbina. Posteriormente se varían los paramentos geométricos hasta encontrar la configuración más adecuada que logre obtener el coeficiente máximo de potencia en función de la solidez. Finalmente se diseña un prototipo para la respectiva impresión en 3D.

\section{Materiales y métodos}

Para evaluar el desempeño de los rotores Darrieus del tipo hidrocinética se utilizan las mismas técnicas que se usan para los rotores eólicos debido a que en ambos casos se consideran ambos fluidos aire y agua como incompresibles, en este trabajo se opta por utilizar el método propuesto por Ion Parashivoiu [1]

*Autor de correspondencia.

E-mail: carloshumbertoap@ufps.edu.co (Carlos Acevedo Peñaloza),

(c) (i) (s) $\odot$ Peer review is the responsibility of the Universidad Francisco de Paula Santander c) 1 (1) $\mathrm{El}$. 
Como se aprecia en la Figura 1, la unidad generadora de electricidad está compuesta por: rotor, engranajes, rodamientos, generador eléctrico, acondicionamiento de potencia y unidad de carga final.

El perfil aerodinámico es quizá el elemento más importante del rotor, ya que, es el encargado de recibir la energía provista por el fluido. Su forma hace que el agua al atravesarla genere un diferencial de presión entre la parte superior e inferior por

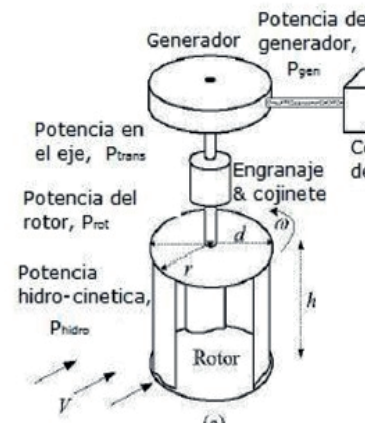

(a)

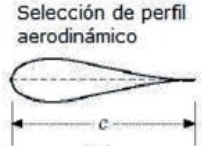

(b)
Figura 1. Unidad generadora Fuente: Khan [5]

causa de la diferencia de velocidades en ambas caras. Para evaluar el perfil más apropiado se aplica el modelo DMST aplicado a turbinas hidrocinéticas como se detalla en el trabajo de Rolón [6]. Gracias a la versatilidad de este modelo es posible analizar otros parámetros geométricos de importancia como el diámetro, cuerda, envergadura y número de álabes.

La variación de todos estos parámetros se realiza con el fin de encontrar una configuración que permita extraer la mayor cantidad de potencia del fluido para posteriormente ser convertida en energía mecánica.

La potencia del rotor está definida por:

\section{Donde:}

$$
P=\frac{1}{2} \rho S_{b} U_{\infty}^{3} C p
$$

$P=$ potencia $(W)$

$\rho=$ densidad $(\mathrm{Kg} / \mathrm{m} 3)$

$\mathrm{Sb}=$ Área de barrido $(\mathrm{m} 2)$

$U \infty=$ Velocidad de flujo libre

El coeficiente de potencia $\mathrm{Cp}$ es un valor adimensional que cuantifica la cantidad de energía que logra extraer el rotor del fluido. Albert Betz estimó cual es el máximo de energía convertible por un rodete estableciendo un límite del 59\% para turbinas eólicas, sin embargo en la práctica estos dispositivos convierten alrededor del $40 \%$.

El coeficiente de potencia también puede ser definido con dos relaciones adimensionales $\lambda$ (razón de velocidades de punta) y $\mathrm{Ct}$ (coeficiente de torque). $\lambda$ es comúnmente utilizado para representar la curva característica del rotor mostrando el $\mathrm{Cp}$ función de este, en varios puntos de operación. La Figura 2, ilustra la relación entre $\mathrm{Cp}$ vs $\lambda$.

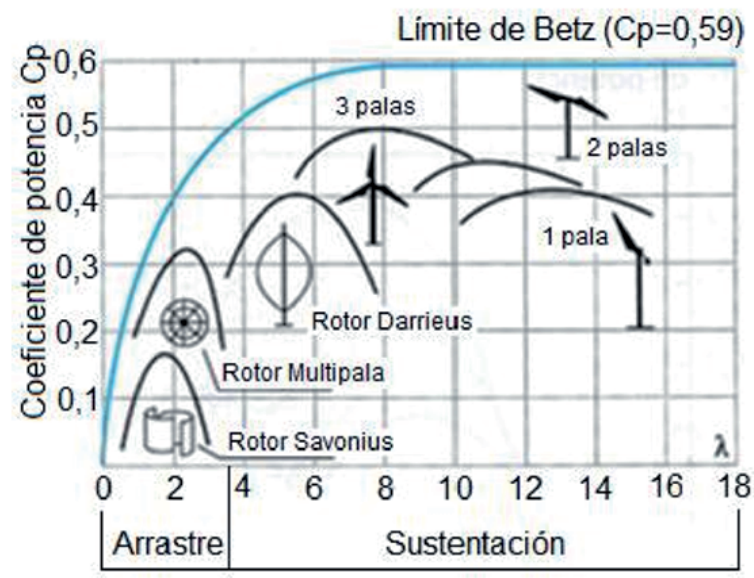

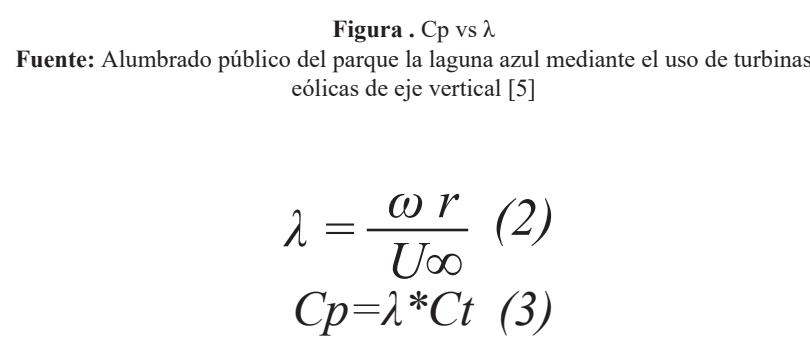

Donde:

$\lambda=$ Razón de velocidades de punta

$\omega=$ Velocidad angular

$C t=$ Coeficiente de torque

Como se señaló al principio, el objetivo es hallar una configuración óptima de rotor Darrieus tipo $\mathrm{H}$, por lo cual se analizaron varios perfiles aerodinámicos por el método DMST; los perfiles estudiados son recopilados de los trabajos realizados por Shiono [3] , M.C. Claessens [6], Khan [5].

Para realizar las simulaciones se prueba con las configuraciones añadidas en la Tabla I. Como se observa en la Figura 3, el perfil DU 06-W-200 es el que tiene las características más adecuadas desde el punto de vista de desempeño, pues registra un coeficiente de potencia de 0.55 en $\lambda=3$., que sumado con un rango de operación amplio permite que con las variaciones de velocidad de flujo la potencia no se vea afectada.

Estos datos fueron tomados según el trabajo de Thomaz [8] con el fin de verificar la capacidad del modelo. Teniendo el perfil seleccionado se procede a buscar las relaciones geométricas más eficientes. La solidez $\sigma$ es un parámetro geométrico que se define como la relación entre: el número de palas, la longitud y el diámetro del rodete. 
Tabla I. Configuración de simulación electrónica

\begin{tabular}{|c|c|}
\hline Parámetro & Magnitud \\
\hline $\begin{array}{c}\text { Velocidad de } \\
\text { entrada }\end{array}$ & $1 \frac{\mathrm{m}}{\mathrm{S}}$ \\
\hline Presión de trabajo & $1 \mathrm{~atm}$ \\
\hline Radio & $3 \mathrm{~m}$ \\
\hline Tipo de perfil & $\begin{array}{c}\text { NACA } 0018, \\
\text { NACA0021, } \\
\text { NACA0015, S1223, } \\
\text { Fx63-137.S815, DU } \\
06-\mathrm{W}-200\end{array}$ \\
\hline Número de alabes & $3 \mathrm{~m}$ \\
\hline Cuerda & $0.25 \mathrm{~m}$ \\
\hline Longitud del alabe & $3 \mathrm{~m}$ \\
\hline $\begin{array}{c}\text { Viscosidad } \\
\text { cinemática }\left(20^{\circ} \mathrm{C}\right)\end{array}$ & $1.007 \mathrm{e}-6 \frac{\mathrm{m}}{\mathrm{s}}$ \\
\hline Densidad $\left(20{ }^{\circ} \mathrm{C}\right)$ & $998.2 \frac{\mathrm{Kg}}{\mathrm{m}^{3}}$ \\
\hline
\end{tabular}

Fuente: Rolón [6]

\section{$\mathrm{Cp} \operatorname{Vs} \lambda$}

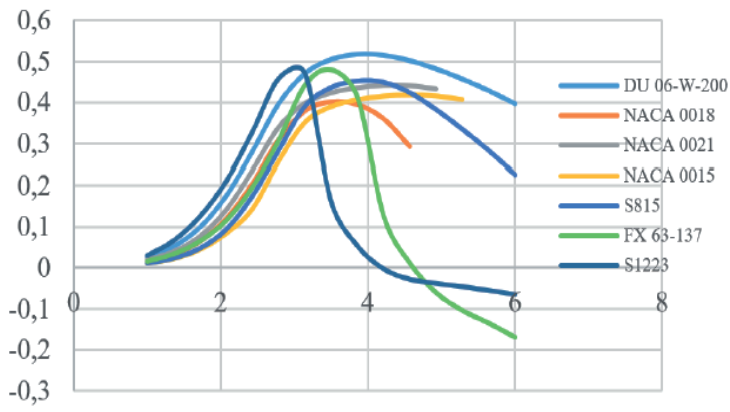

Figura 3. Comparación de perfiles de estudio $\lambda$ vs $\mathrm{Cp}$ Fuente: Rolón [6]

$$
\sigma=\frac{N^{*} l}{c}(4)
$$

\section{Donde:}

$N=$ número de palas

$l=$ longitud de la pala

$C=$ longitud de la cuerda

Se procedió a correr de nuevo el programa esta vez variando la solidez con el perfil seleccionado. Como se observa en la Figura 4, la solidez influye de manera importante en el desempeño de la turbina. Según los resultados, la solidez óptima para esa configuración es de aproximadamente 0.55 .

\section{Consideraciones de diseño}

Para diseñar un rodete se debe considerar primero la potencia de salida requerida. En este trabajo se definió una potencia requerida de $28 \mathrm{~W}$ definida en la ecuación (Eq. ( 5 )) Con el fin

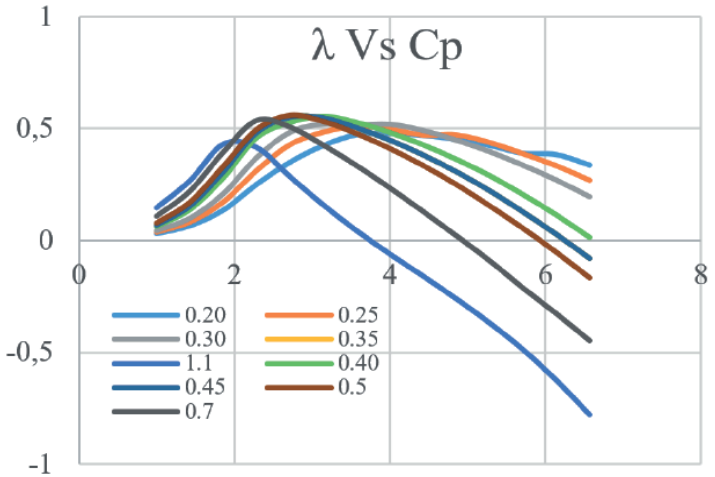

Figura 4. Influencia de la solidez en el desempeño Fuente: Rolón [6]

de alimentar en teoría una bombilla actuadora. Como segundo parámetro importante se deben contar con estudios de la velocidad de flujo donde se va a ubicar la turbina, en este caso se tomó una velocidad promedio de $1 \mathrm{~m} / \mathrm{s}$ como velocidad de diseño.

Se deben tener en cuenta las pérdidas por los elementos que componen el sistema de transmisión: engranes, generados, etc. Estos elementos tienen una eficiencia aproximada del $88 \%$ según datos tomados de Khan [5]. La densidad del agua depende de la temperatura, en este caso se tomó una temperatura de

$$
P=n s y s \frac{1}{2} \rho S_{b} U_{\infty}^{3} C p \text { (6) }
$$

operación promedio de $25^{\circ} \mathrm{C}$ como se define en la ecuación Eq. (6), la potencia también depende del área de barrido $S b$, teniendo en cuenta la solidez óptima definida en el apartado anterior, se asume un valor de cuerda y se despeja de este el diámetro y la longitud del perfil, para este estudio se tomó una longitud de cuerda de $0.025 \mathrm{~m}$ y 3 aspas, posteriormente se despejo de la ecuación de área de barrido $S b$ el diámetro de del rodete.

$$
S b=l * d \text { (7) }
$$

Donde:

$d=$ diámetro del rotor

Teniendo la geometría definida como se muestra en la Tabla II, se realizó la respectiva simulación en CFD con el fin de validar dichos resultados. 


\section{Simulación en ANSYS FLUENT}

Para realizar la simulación es necesario crear el dominio a estudiar, en este caso se usa la metodología propuesta por la Universidad de Cornell [9] usando un dominio redondo concéntrico al rodete.

Tabla II. Dimensiones geométricas
\begin{tabular}{|l|l|}
\hline Parámetro & Magnitud \\
\hline $\mathrm{P}(\mathrm{W})$ & 28 \\
\hline Cuerda $(\mathrm{m})$ & 0,025 \\
\hline Diámetro $(\mathrm{m})$ & 0,296 \\
\hline Altura $(\mathrm{m})$ & 0,296 \\
\hline \multicolumn{2}{|c|}{ Fuente: Rolón [6] }
\end{tabular}

Desarrollo de malla: Teniendo ya la geometría se procedió a realizar la malla en ANSYS ICEM 16.0 ICEM crea bloques que están compuestos por ejes y vértices, estos a su vez son asociados a la geometría, la creación de los bloques permite dar características especiales a la malla en secciones donde se requiere enmallar de manera más precisa como se muestra.

Luego se procede a la división de los ejes en el número de elementos requeridos, diferenciando el alabe donde se tendrá un tratamiento especial usando una malla tipo $\mathrm{O}$ también conocida O-grid, lo que significa que las líneas de malla siguen un patrón ordenado, encerrando la geometría siguiendo un patrón concéntrico.

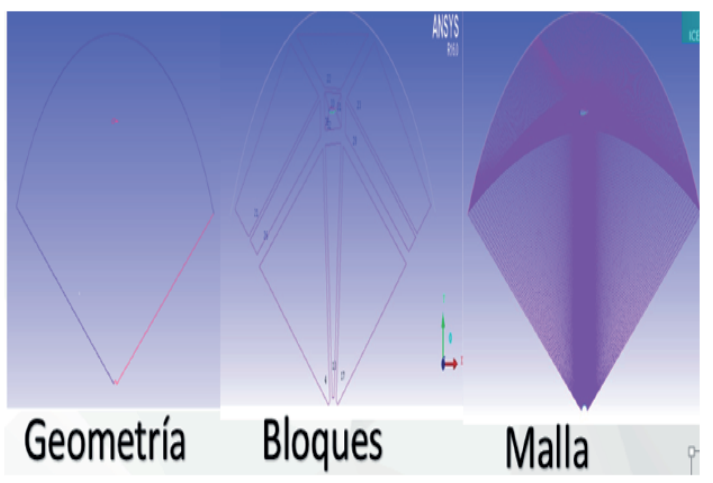

Figura 5. Procedimiento de enmallado Fuente: Rolón [6]

Importada la malla, se configura la salida de datos de resultados mediante el solution setup donde se selecciona el modelo de turbulencia; en este caso se utilizó k-omega SST debido a que se necesita gran precisión en la resolución de capa limite, ya que el perfil presenta separación de flujo, altos gradientes de presión y flujo inverso. Fluent utiliza el modelo k- $\omega$ en la región de la capa límite, y progresivamente cambia al modelo k-E a medida que se aleja de la superficie de capa límite usando lo que se conoce como bending function. Esto logra mantener el superior tratamiento de pared el modelo k- $\omega$, eliminando su dependencia del flujo lejos de la pared.

\section{Resultados y análisis}

Como se observa en las Figuras 6 y t7, los resultados de la simulación y el modelo matemático tienen buena correlación por lo cual se consideran validados.

Este comportamiento es similar a las simulaciones de turbinas hidrocinéticas realizadas por S. Lain [10] donde la curva CFD estuvo por encima de la DMST tomando en cuenta las evaluaciones realizadas se realiza un modelo en Solidworks para su posterior impresión.

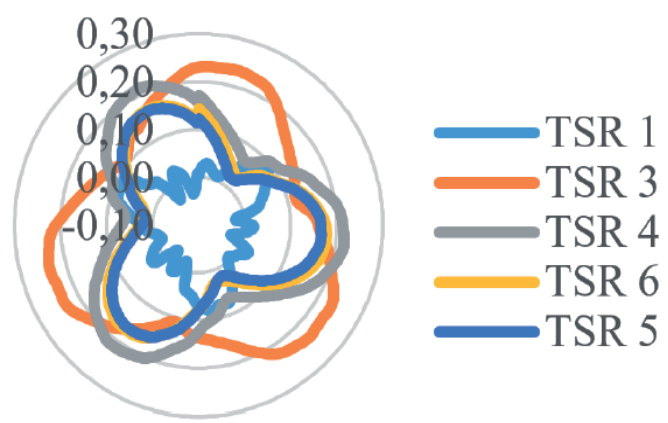

Figura 6. Coeficiente de momento vs ángulo de rotación Fuente: Rolón [6]

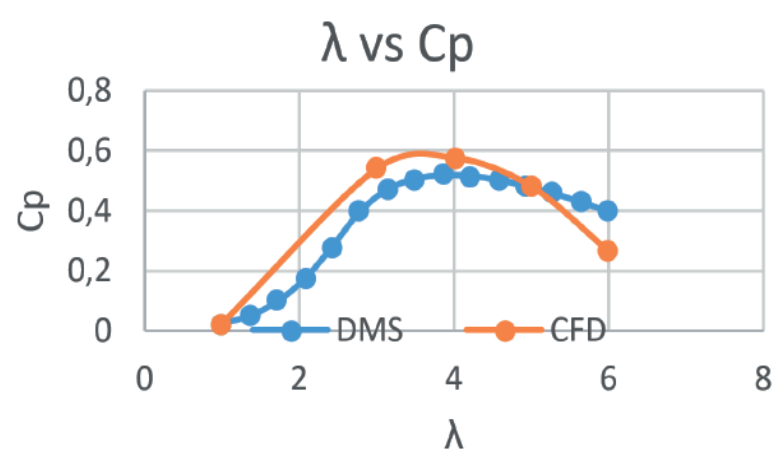

Figura 7. $\lambda \mathrm{Vs} \mathrm{Cp}$

Fuente: Rolón [6]

\section{Conclusiones}

Partiendo del análisis se encontró que el perfil DU 06-W-200 es el que tiene mejores prestaciones al momento de extraer energía, además cuenta con un rango de funcionamiento más amplio resistiendo cambios en las condiciones de operación como en la velocidad del flujo, afectando al mínimo el rendimiento. Este perfil combinado con un grado de solidez de 0.55 entrega un coeficiente de potencia de 0.56 .

El material del rodete es fundamental para garantizar su vida útil, debido a las condiciones hostiles de operación en un entorno natural como por ejemplo en un rio o quebrada. 

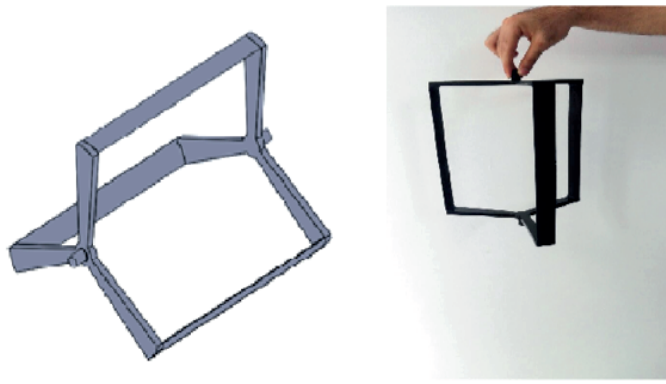

Figura 8. Esquemas del rodete en Solidworks e impresión 3D Fuente: Rolón [6]

Por otro lado, como lo muestra la Figura 7, el torque sufre fuertes variaciones durante la rotación y esto debe ser soportado por el eje y los alabes, por lo tanto se deben usar materiales con alta resistencia a la fatiga y bajo peso.

\section{Referencias}

[1] I. Paraschivoiu, "Wind turbine design with emphasis on darrieus concept" [Diseño de turbina eólica con énfasis en concepto darrieus], P. Polytechnique, Ed., Montréal: 4, 2009.

[2] R. E. Sheldahl y P. C. Klimas, "Aerodynamic characteristics of seven symmetrical airfoil sections through 180-degree angle of attack for use in aerodynamic analysis of vertical axis wind turbines", México: Board, 1981.

[3] M. S. K. \&. K. S. Shiono, "An Experimental Study of the Characteristics of a Darrieus Turbine for Tidal Power Generation [Un estudio experimental de las características de una turbina Darrieus para marea generacion de energia]", Electrical Engineering in Japan, vol. 132, no. 3, pp. 38-47, 15 Junio 2000 .

[4] A. Malipeddi and D. Chatterjee, "Influence of duct geometry on the performance of Darrieus hydroturbine" [Influencia de la geometría de los conductos en el rendimiento de la hidroturbina Darrieus], India: Board, 2012.

[5] M. J. Khan, M. T. Iqbal y J. E. Quaicoe, "Design Considerations of a Straight Bladed Darrieus Rotor for River Current Turbines [Consideraciones de diseño de un rotor Darrieus de hoja recta para turbinas de corriente del río]" IEEE International Symposium on Industrial Electronics, 2006.

[6] H. Rolón O y Y. Villamizar G, "Modelamiento y simulación del comportamiento de rodete tres", Cúcuta, Colombia, 2017.

[7] M. Claessens, "The Design and Testing of Airfoils for Application in Small Vertical Axis Wind Turbines" [El diseño y prueba de superficies aerodinámicas para su aplicación en turbinas eólicas de eje vertical pequeño], Delft, 2006.
[8] T. J. CELSO RANGE, "Desempeño aerodinámico de turbinas eólicas de eje vertical en función de temperatura de superficie de álabe". Santiago de Chile, 2012.

[9] Confluence.cornell.edu, "FLUENT Learning Modules SimCafe - Dashboard," Universidad Cornell, [En línea]. Available: https:/confluence.cornell.edu/display/SIMULATION/FLUENT+Learning+Modules. [Accedido en: 13 Mayo 2017].

[10] S. Lain and C. Osorio, "simlation and evaluation of a straight bladed Darrieus type cross flow marine turbine[Simulación y evaluación de una turbina marina de flujo cruzado tipo Darrieus de pala recta]," Journal of scientific \& insdustrial Research, vol. 69, pp. 906-912, 2010. 